IV.

Die praktische Verwendung der Resultate ist evident. Man kann in sehr vielen Fällen das Destillationsverhalten von Gemischen durch Zusätze bessern bzw. nicht trennbare Gemische überhaupt erst durch Destillation trennfähig machen. Hierunter fallen auch die bekannten Beispiele ,azeotroper" Destillation.

Für die praktische Ausführung hat man dafür zu sorgen, daß der Zusatz in der gewünschten Konzentration in der Destillationskolonne anwesend ist. Je nach dem Siedeverhalten des $\mathrm{Zu}$ satzes erreicht man dies durch Aufgeben an einer geeigneten Stelle der Kolonne, in der Blase oder im Kondensator. Die erwähnten Systeme mit Anilin ergaben z. B. ausgezeichnetes Destillationsverhalten, wenn auf eine kontinuierlich arbeitende Kolonne in einigem Abstand unter dem Kopf Anilin zulaufen gelassen wurde. Die leichter siedende Komponente konnte dann rein ge- wonnen werden, die andere mußte in einer zweiten Kolonne vom Anilin getrennt werden. Aus dem sonst durch Destillation untrennbaren Gemisch Cyclohexan-Benzol, das sich in einer sehr großen Blase befand, konnte fast reines Cyclohexan abdestilliert werden, indem in einer diskontinuierlich arbeitenden Kolonne in der angegebenen Weise Anilin zugegeben wurde, das sich in der Blase anreicherte.

\section{V.}

Weiteres Material soll später an anderer Stelle publiziert werden. Die Untersuchung aller in Frage kommenden thermodynamischen Größen an einer Reihe binärer und tertiärer Systeme ist in Angriff genommen worden.

Die voranstehend beschriebenen Ergebnisse wurden während der Jahre 1941 bis 1943 bei dem Versuch der Reindarstellung reaktionskinetisch interessierender Substanzen erhalten.

\title{
Über die Hydrolyse einiger organischer Halogenverbindungen in Dioxan-Wasser-Gemischen
}

\author{
Von Horst Böhme und Hans-Jürgen Henning \\ Aus dem Kaiser-Wilhelm-Institut für physikalische Chemie und Elektrochemie, Berlin- \\ Dahlem ${ }^{1}$ \\ (Z. Naturforschg. 1, 580-584 [1946]; eingegangen am 20. Juli 1946)
}

\begin{abstract}
Die Hydrolyse von Benzylchlorid, Methyl-phenyl-chlor-methan und Diphenyl-chlormethan wird in Wasser-Dioxan-Gemischen verschiedener Zusammensetzung gemessen. Es wird eine starke Abhängigkeit der Reaktionsgeschwindigkeit vom Wassergehalt des Mediums festgestellt und darüber hinaus eine Beziehung zwischen den Geschwindigkeitskonstanten und den Dielektrizitätskonstanten der Dioxan-Wasser-Gemische wahrscheinlich gemacht, die sich mathematisch formulieren läßt.
\end{abstract}

$\mathrm{B}$ ei der Messung der Hydrolysegeschwindigkeit organischer Halogenverbindungen ist das chemisch recht indifferente und mit Wasser in jedem Verhältnis mischbare Dioxan als Lösungsmittel sehr geeignet, weil es die Durchführung der Reaktion im homogenen Medium erlaubt. Bei den bisherigen Veröffentlichungen ${ }^{2}$ handelt es sich nun meist um spezielle Fragestellungen, während die Abhängigkeit der Reaktionsgeschwindigkeit

1 Derzeitige Anschrift des Verf.: Pharmazeutischrhemisches Institut der Universität Marburg (Lahn). von der Zusammensetzung der Dioxan-WasserGemische bisher noch nicht auf breiterer Basis untersucht wurde. Eine Klärung dieser Frage wurde wünschenswert im Verlauf der vor einiger Zeit durchgeführten Untersuchung ${ }^{3}$ sauerstoffund schwefelhaltiger Halogenverbindungen, deren Hydrolyse in wechselnd zusammengesetzten Di-

2 A. R. Ol sen u. R. S. H a l f o r d, J. Amer. chem. Soc. 59, 2644 [1937]; D. R. R e a d u. W. T a y l o r, J chem. Soc. [London] 1939, 478; G. W. B e s te u L. P. H a m m e t, J. Amer. chem: Soc. 62, 2481 [1940]. ${ }_{3}$ H. B ö h m e. Ber. dtsch. chem. Ges. 74, 248 [1941] 
oxan-Wasser-Gemischen gemessen wurde. Hierbei war die Frage offen geblieben, ob die bei gröBeren Wasserkonzentrationen gemessenen höheren Hydrolyse-Geschwindigkeiten allein auf die damit verbundene Steigerung der Umsetzungsmöglichkeit zurückzuführen ist, oder ob daneben noch andere Faktoren wie die Änderung der Dielektrizitätskonstanten (D.K.) eine Rolle spielen. Bei Umsetzungen, die als Kryptoionenreaktionen im Sinne von H. M e erwein verlaufen, war nämlich durch Untersuchung in Lösungsmitteln mit verschiedener D.K. eine Abhängigkeit der Reaktionsgeschwindigkeit von den dielektrischen Eigenschaften des Lösungsmittels schon früher wahrscheinlich gemacht worden ${ }^{4}$. Da das System Wasser-Dioxan im Hinblick auf diese Eigenschaften sehr gründlich untersucht ist ${ }^{5}$ und hier die D.K. in durchsichtiger Weise vom Mischungsverhältnis der beiden Komponenten abhängt, schien eine Möglichkeit gegeben, die Geschwindigkeit der Hydrolyse bei verschiedener D.K. des Mediums messen zu können, wobei aber im Gegensatz zu den früheren Versuchen nicht nur eine lückenlose Reihe von Medien verschiedener D.K. realisierbar ist, sondern darüber hinaus aúch die durch verschiedenen chemischen Bau der Lösungsmittel bedingten speziellen Einflüsse ausgeschaltet sind. Wir haben daher die Hydrolyse einiger einfacher Halogenverbindungen in Dioxan-Wasser-Gemischen verschiedener Zusammensetzung untersucht.

Das relativ reaktionsträge Benzylchlorid wurde bei $100^{\circ}$ und hohem Wasserüberschuß hydrolysiert. Abb. 1 zeigt die Geschwindigkeitskonstanten der nach der I. Ordnung verlaufenden Reaktion, aufgetragen gegen die Mole Wasser, die auf $1 \mathrm{Mol}$ Halogenverbindung in einem Wasser-Dioxan-Gemisch konstanter Zusammensetzung (30,0\% Wasser) kommen ${ }^{6}$. Die Kurve gibt also lediglich die Abhängigkeit der Reaktionsgeschwindigkeit von dem verschiedenen molaren Verhältnis Halogenverbindung: Wasser wieder. Dieser Einfluß ist relativ gering, die Reaktionsgeschwindigkeitskonstante wächst um etwa $50 \%$ bei einer Verzehnfachung des molaren Wasserüberschusses.

Ganz anders liegen die Verhältnisse aber, wenn

4 Z. B. H. M e e rw e in u. K. van Emster, Ber. dtsch. chem. Ges. 55, 2500 [1922]; K. B o d e n d o r f u. H. B ö h m e, Liebigs Ann. Chem. 516, 1 [1935].

- G. A k e r l ö f u. O. A.S h or t, J. Amer. chem. Soc. 58, 1241 [1936]. bei gleichbleibendem molaren Verhältnis von Halogenverbindung: Wasser die Hydrolyse im verschieden zusammengesetzten Dioxan-Wasser-Gemisch gemessen wird. In Abb. 2 sind die bei Verwendung von $200 \mathrm{Mol}$ Wasser auf $1 \mathrm{Mol}$ Halogenverbindung erhaltenen Reaktionsgeschwindigkeits konstanten I. Ordnung gegen den jeweils ange-

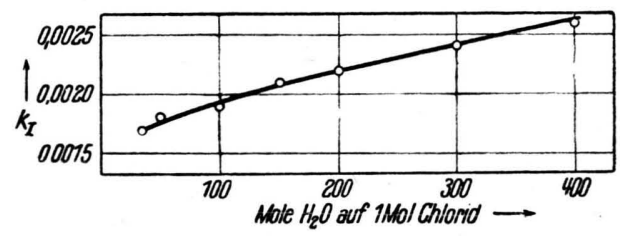

Abb. 1. Hydrolyse von Benzylchlorid bei $100^{\circ}$ in einem 30,0-proz. Wasser-Dioxan-Gemisch. Abhängigkeit der Reaktionsgeschwindigkeit vom mol. Wasserüberschuß

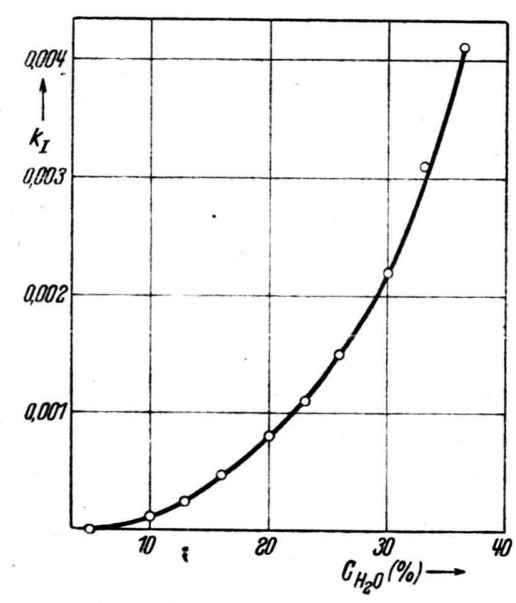

Abb. 2. Hydrolyse von Benzylchlorid bei $100^{\circ}$ und kon stantem mol. Wasserüberschuß $(1: 200)$. Abhängigkeit der Reaktionsgeschwindigkeitskonstanten vom proz Wassergehalt des Mediums.

wandten prozentualen Wassergehalt des Lösungs mittelgemisches aufgetragen. Man erkennt ein starkes Anwachsen der Geschwindigkeitskonstanten in Abhängigkeit vom Wassergehalt des $\mathrm{Me}$ diums; eine Steigerung der Wasserkonzentration um etwa das 7-fache bewirkt eine Erhöhung der Konstanten auf einen über 200-mal größeren Wert. Von besonderem Interesse ist nun, daß man beim Auftragen der Logarithmen der Reaktionsgeschwindigkeitskonstanten gegen die Logarith-

6 B este u. H a m met ${ }^{2}$ haben ähnliche Versuche bei $50^{\circ}$ und einer anderen Zusammensetzung des Dioxan-Wasser-Gemisches bereits durch geführt und ähnliche Ergebnisse erzielt. 
men der Wasserkonzentrationen eine Gerade erhält (Abb.3).

Ähnliche Verhältnisse fanden wir auch bei den sekundären Chloriden Methyl-phenyl-chlor-methan und Diphenyl-chlor-methan. Diese Verbindungen sind wesentlich reaktionsfähiger als Benzylchlorid, so daß entsprechende Messungen auch bei tieferen Temperaturen durchgeführt werden konnten. Es finden sich in Abb. 3 für die verschiedenen Reaktionen die Logarithmen der Reaktionsgeschwindigkeitskonstanten gegen die Logarithmen

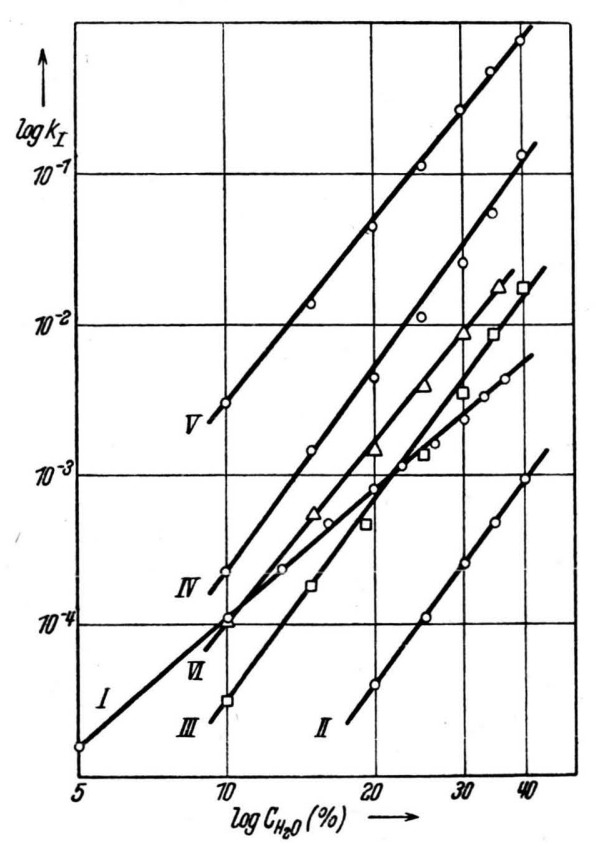

Abb. 3. Hydrolyse bei konstantem mol. Wasserüberschuß (1:200) von Benzylchlorid bei $100^{\circ}$ (I), Methylphenyl-chlor-methan bei $25^{\circ}$ (II), bei $50^{\circ}$ (III), bei $70^{\circ}$ (IV), bei $100^{\circ}$ (V), von Diphenyl-chlor-metan bei $25^{\circ}$ (VI). Abhängigkeit der Reaktionsgeschwindigkeitskonstanten vom proz. Wassergehalt (logarithmisch dargestellt).

der Wassergehalte des Mediums aufgetragen. In allen Fällen ist also das molare Verhältnis von Halogenverbindung : Wasser wie 1 : 200. Die Messungen mit Methyl-phenyl-chlor-methan bei vier verschiedenen Temperaturen (25, 50, 70 und $100^{\circ}$ ) werden in der doppelt logarithmischen Darstellung durch vier annähernd parallel liegende Gerade wiedergegeben ${ }^{7}$.

7 Die Temperaturabhängigkeit der Geschwindig. keitskonstanten folgt der A r r hen i s schen Gleichung. Die dekadischen Temperaturkoeffizienten schwanken zwischen 1,8 und 3,0 .
Versuche mit dem sehr reaktionsfähigen Triphenyl-chlor-methan sind insofern weniger übersichtlich, als die Reaktion bei dem hier erforderlichen geringen Wasserüberschuß zu einem Gleichgewicht führt. Diese Befunde sollen noch ergänzt werden; sie zeigen aber gleichfalls einwandfrei, daß auch hier die Reaktionsgeschwindigkeit in erster Linie von der Zusammensetzung des Dioxan-Wasser-Gemisches abhängt, während die Lage des Gleichgewichtes vor allem durch das Verhältnis der molaren Konzentrationen der beiden Reaktionsteilnehmer bestimmt wird.

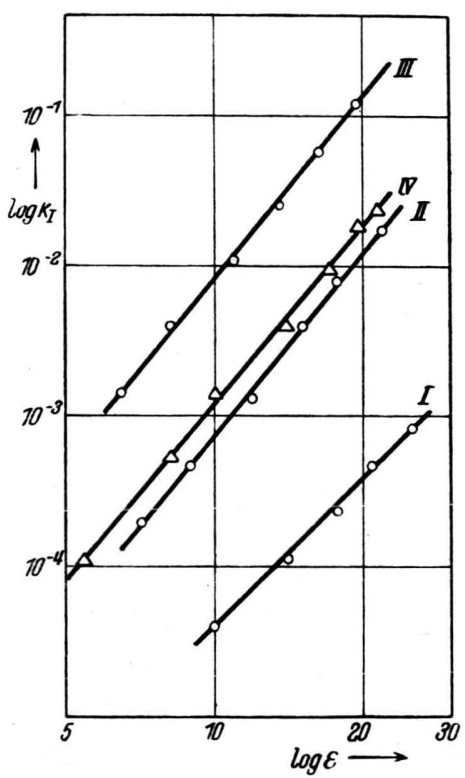

Abb. 4. Hydrolyse bei konstantem mol. Wasserüberschuß $(1: 200)$ von Methyl-phenyl-chlor-methan bei $25^{\circ}$ (I), bei $50^{\circ}$ (II), bei $70^{\circ}$ (III), von Diphenyl-chlormethan bei $25^{\circ}$ (IV). Abhängigkeit der Reaktionsgeschwindigkeitskonstanten von der Dielektrizitätskonstanten des Mediums (logarithmisch dargestellt).

Die gefundene starke Abhängigkeit der Hydrolysegeschwindigkeit der untersuchten Halogenverbindungen von der Zusammensetzung des Dioxan-Wasser-Gemisches kann als Hinweis darauf angesehen werden,- daß die Dielektrizitätskonstante des Mediums eine wichtige Rolle bei der Reaktion spielt. Es ist in diesem Zusammenhang von Interesse, daß man gleichfalls eine Gerade erhält, wenn man an Stelle der Logarithmen der prozentualen Zusammensetzung des Dioxan-Wasser-Gemisches die der zugehörigen Dielektrizitäts- 


\begin{tabular}{|c|c|c|}
\hline Min. & ccm 0,1-n. KOH & $k_{I}$ \\
\hline & 0,59 & \\
15 & 2,32 & 0,00163 \\
64 & 4,54 & 0,00170 \\
128 & 10,92 & 0,00175 \\
364 & 12,65 & $0,(10153$ \\
513 & 15,52 & 0,00187 \\
593 & 21,25 & 0,00172 \\
1434 & 23,22 & - \\
ber. Endwert & & \\
& & \\
\hline
\end{tabular}

$k_{I}$ (im Mittel) 0,0017

Tab. 1. Benzylchlorid / $100^{\circ}$ C; 3,008 g Sbst., 15,0 g Wasser, 35,0 g Dioxan; 30,0 Gew.-\% Wasser im Lösungsmittel. $5 \mathrm{ccm}$ Reaktionsgemisch wiegen $5,18 \mathrm{~g}$. Sbst.: 0,464-mol., Wasser: 16,3-mol. (Verh. $1: 35,0)$.

\begin{tabular}{|c|c|c}
\hline Min. & ccm 0,1-n. KOH & $k_{I}$ \\
\hline 15 & 0,13 & $-\overline{13}$ \\
$\mathbf{6 3}$ & 0,35 & 0,00240 \\
126 & 0,60 & 0,00239 \\
361 & 1,36 & $0,(10271$ \\
591 & 1,72 & 0,00269 \\
ber.Endwert & 2,15 & - \\
\hline
\end{tabular}

$$
k_{I} \text { (im Mittel) } 0,0026
$$

Tab. 2. Benzylchlorid $/ 100^{\circ} \mathrm{C} ; 0,265 \mathrm{~g}$ Sbst., $15,0 \mathrm{~g}$ Wasser, 35,0 g Dioxan; 30,0 Gew.-\% Wasser im Lösungsmittel. 5 ccm Reaktionsgemisch wiegen $5,15 \mathrm{~g}$. Sbst.: 0,0429-mol.. Wasser: 17,1-mol. (Verh. 1: 397).

konstanten ${ }^{\triangleleft}$ gegen die Logarithmen der Reaktionsgeschwindigkeitskonstanten aufträgt (Abb. 4). Beide Größen sind also miteinander durch die Beziehung

$$
\begin{aligned}
\log k & =m \cdot \log \varepsilon+\log n \\
k & =n \cdot \varepsilon^{m}
\end{aligned}
$$

verknüpft, wobei die Werte für $m$ und $n$ aus den Neigungen der Geraden bzw. den Ordinatenabschnitten entnommen werden können.

Unsere Befunde, die zu weiteren Versuchen anregen und nach den verschiedensten Richtungen ergänzt und erweitert werden sollen, lassen sich also zwanglos durch die Abhängigkeit der Reaktionsgeschwindigkeit von der D.K. des Mediums deuten. Diese Tatsache kann gleichzeitig als Stütze für die Auffassung dieser Hydrolysen als Kryptoionenreaktionen angesehen werden. Dafür spricht

8 Berechnet aus den Angaben von Akerlö f $u$. Sh o t $^{5}$. Die durch die im Verlauf der Reaktion gebildeten Hydrolyseprodukte bedingte kontinuierliche Änderung der Dielektrizitätskonstanten blieb dabei zunächst unberücksichtigt.

\begin{tabular}{|c|c|c|}
\hline Min. & ccm $0,1-n . \mathrm{KOH}$ & $k_{I}$ \\
\hline 14 & 0,02 & - \\
78 & 0,03 & 0,000112 \\
576 & 0,11 & 0,000116 \\
1728 & 0,25 & 0,000103 \\
4554 & 0,59 & 0,000113 \\
5810 & 0.66 & 0,000103 \\
7550 & 0,85 & 0,000117 \\
10010 & 0,98 & 0,000113 \\
ber. Endwert & 1,44 & - \\
\hline \multicolumn{2}{|c|}{$k_{I}$ (im Mittel) 0,00011} \\
\hline
\end{tabular}

Tab. 3. Diphenyl-chlor-methan $/ 25^{\circ} \mathrm{C} ; 0,284 \mathrm{~g}$ Sbst.. 5,00 g Wasser, 45,0 g Dioxan; 10,0 Gew.-\% Wasser im Lösungsmittel. 5 cem Reaktionsgemisch wiegen $5,17 \mathrm{~g}$ Sbst.: 0,0288-mol., Wasser: 5,71-mol. (Verh. 1:198).

\begin{tabular}{|c|c|c|}
\hline Min. & ccm 0,1-n. KOH & $k_{I}$ \\
\hline 1 & 2,87 & - \\
2 & 4,31 & 0,737 \\
3 & 5,01 & 0,747 \\
4 & 5,33 & 0,740 \\
ber. & 5,63 & - \\
Endwert & & \\
\hline \multicolumn{2}{|l|}{$k_{I}$ (im Mittel) 0,74} \\
\hline
\end{tabular}

Tab. 4. Meihyl-phenyl-chlor-methan $/ 100^{\circ}$ C; $0,784 \mathrm{~g}$ Sbst., 20,0 g Wasser, 30,0 g Dioxan; 40,0 Gew.-\% Wasser im Lösungsmittel. 5 ccm Gemisch wiegen $5,13 \mathrm{~g}$ Sbst.: 0,113-mol., Wasser: 22,4-mol. (Verh. 1: 199).

auch, daß man bei Verwendung von optisch aktiven Phenyl-methyl-chlormethan eine nach der I. Ordnung verlaufende vollständige Racemisierung beobachtet, die in allen untersuchten Fällen mit derselben Geschwindigkeit verläuft wie die titrimetrisch gemessene Hydrolyse.

\section{Beschreibung der Versuche}

Die verwendeten Halogenverbindungen wurden nach den im Schrifttum angegebenen Verfahren dargestellt und durch mehrmalige Destillation über eine $\mathrm{Wid}$. me r-Kolonne gereinigt. Das als Lösungsmittel benutzte Dioxan wurde nach den Angaben von $\mathrm{E}$. E i g e $\mathrm{n} b$ e $\mathrm{r}$ g e $\mathrm{r}^{9}$ und $\mathrm{R}$. L e i m u ${ }^{10}$ gereinigt.

Zur Durchführung der Messung wurde eine genau gewogene Menge der Halogenverbindung in der be rechneten Gewichtsmenge Dioxan gelöst und mit der berechneten, meist volumetrisch abgemessenen Menge Wasser versetzt. Im allgemeinen waren diese Mengen so gewählt, daß von insgesamt etwa $50 \mathrm{~g}$ Reaktionsgemisch ausgegangen wurde, von denen je $5 \mathrm{ccm}$ für

9 J. prakt. Chem. (2) 130, 78 [1931].

10 Ber. dtsch. chem. Ges. 70, 1040 [1937]. 